recombination intervals (the origin and terminus of replication were preferentially involved) and $r e c$ functions (recA-independent recombination) $(3,11,15,16)$.

The so-called $\mathbf{C D}$ (complementing diploids) were selected as prototrophs due to the complementation of several allelic mutations carried by the parental strains; they were by far the most unstable, and stabilized rapidly as prototrophic or auxotrophic recombinants of the $\mathrm{R}$ type [with recombinations taking place in the above-mentioned preferential intervals (10)]. These recombinants were named secondary, in contrast with the directly selected, primary recombinants. The presence of a dominant $s p o 0 \mathrm{~A}$ defective allele has been useful in the study of colonial variations in the sporulation phenotypes of prototrophs obtained from a Spo0 $\mathrm{A}^{+} \times \mathrm{SpoOA} \mathrm{A}^{-}$pair (15); only a few of them are still of the CD type when they appear on selective $\mathrm{mR} 2$ regeneration plates. Recently, the presence of this mutation was shown to increase the duration of instability of NCD (9).

(ii) Abundant experimental data support the diploidy of all the fusion types. However, only CD cells can be considered as true diploids, according to criteria based on physiological (complementation), genetical and biochemical approaches (identical recovery of both alleles by transformation); they are, however, highly unstable and rapidly lead to recombinants (10). The rest of the progeny recombinants, and NCD, are diploids of a novel type in prokaryotic genetics, with several inactive alleles or an entire inactive chromosome. This DNA is poorly accessible to routine genetical and biochemical analyses such as transformation $(1,4)$, hybridization or other techniques applied to detect it (12). In this situation the number and proportion of baploid cells contained in one of any of the fusion products cannot be established; this conclusion has been pointed out in the majority of the previous papers. Every previous worker in this field has searched for the usual signs of diploidy without success: the size of colonies or of cells, the culture generation time, the presence of increased amounts of DNA or of distinctive peaks of nucleoids, did not reveal any unique identifying properties.

Our response. Hauser \& Karamata (6) did not take into account the above considerations. In addition, we feel that they made the following points which were invalid.

(i) They consider the inactive chromosome to be as efficiently accessible as active DNA using the same biochemical techniques whose insensitivity had been shown in the previous papers. They searched for the presence of inactive alleles by quantitative estimations of hybridization. They did not analyse the variations of any colonial phenotype. No novel molecular approach or technique, e.g. PCR, was applied to obtain a novel result.

(ii) While Hauser and Karamata did not determine the number of haploid cells in a clone, they derive conclusions from quantitative determinations of the amount of DNA per cell from only 50 individual cells, obtained from one NCD clone, and the same number of cells from three prototrophic clones, considered by them to be of $\mathrm{CD}$ type only by checking for prototrophy and not from their unstable phenotype. From our experience (10) these CD clones were certainly recombinants.

With respect to the criticisms raised by Hauser and Karamata, let us analyse a previous paper of ours (15) concerning the diploidy of the 'recombinant progeny' or the so-called 'phenotypically recombinant progeny' (15).

Their suggestion that Sanchez-Rivas and coworkers were dealing with syntrophic mixtures cannot be substantiated, since the primary and secondary recombinants described in that paper were all analysed after several purification protocols (re-isolation on minimal medium and sporulation) that are considered acceptable by Hauser and Karamata.

The phenotype of these recombinants cannot be explained by a simple transformation occurring in the plate. In fact such phenotypes could not be understood unless both entire parental chromosomes were in the same cell, because of the intervals involved and the frequencies of recombination. Among the recombinants resulting from the same number of cross-overs, those involving the origin and terminus of replication were 100 times more frequent.

When these recombinants were analysed by transformation, diploidy was demonstrated for eight markers distributed around the chromosome. In addition, although the recombinants were phenotypically $\mathrm{Trp}^{-}$, the inactive wild-type trp allele was also shown to be present.

Of the recombinants obtained from fusions in which only one parent was lysogenic for the $\phi 105$ prophage, all $(40 / 40)$ were lysogenic. The simple explanation of their occurrence by prophage induction is ruled out by the fact that induction of the prophage was not observed during the fusion procedure $(14,16)$.

Regarding the criticism of the poor burstsize reported with NCD clones containing the thermoinducible repressor mutation cts 23 of prophage $\phi 105$ (5), data from this (13) and other laboratories [J. Errington (2), during his construction of $\phi 105$ cloning vectors] indicate that this mutation is poorly inducible.

On the basis of the above comments we feel justified in concluding that the experimental support of Hauser \& Karamata (6) is insufficient to dismiss the polyploidy of the fusion progeny.

Carmen Sanchez-Rivas ${ }^{1 *}$ and Corinne Levi-Meyrueis $^{2}$

${ }^{1}$ Facultad de Ciencias Exactas y Naturales, Dto de Quimica Biologica, Ciudad Universitaria Pabellon II, 1428 Buenos Aires, Argentina.

2 CNRS-CEREMOD, 9 rue Jules Hetzel, 92190 Meudon, France.
* For correspondence. Fax: +54 17433974 . e-mail: sanchez@genbac.edu.ar

1. Bohin, J. P., Ben Khalifa, K., Guillén, N., Schaeffer, P. \& Hirschbein, L. (1982). Phenotypic expression in vivo and transforming activity in vitro: two related functions of folded bacterial chromosomes. Mol \& Gen Genet 185, 65-68.

2. Errington, J. (1984). Efficient Bacillus subtilis cloning system using bacteriophage vector $\phi 105 \mathrm{~J} 9$. J Gen Microbiol 130, 2615-2628.

3. Gabor, M. H. \& Hotchkiss, R. D. (1983). Reciprocal and nonreciprocal recombination in diploid clones from Bacillu. subtilis protoplast fusion: association with the replication origin and terminus. Proc Natl Acad Sci USA 80, 1426-1430. 4. Guillen, N., Gabor, M. H., Hotchkiss, R. D. \& Hirschbein, L. (1982). Isolation and characterization of the nucleoid of non-complementing diploids from protoplast fusion in Bacillus subtilis. Mol ơ Gen Genet 185, 69-74.

5. Guillen, N., Sanchez-Rivas, C. \& Hirschbein, L. (1983). Absence of functional RNA encoded by a silent chromosome in non-complementing diploids obtained from protoplast fusion in Bacillus subtilis. Mol \&o Gen Genet 191, 81-85.

6. Hauser, P. M. \& Karamata, D. (1992). Ploidy of Bacillus subtitis exfusants: the haploid nature of cells forming colonies with biparental or prototrophic phenotypes. J Gen Microbiol 138, 1077-1088.

7. Hotchkiss, R. D. \& Gabor, M. H. (1980). Biparental products of bacterial protoplast fusion showing unequal parental chromosome expression. Proc Natl Acad Sci USA 77 3553-3557.

8. Hotchkiss, R. D. \& Gabor, M. H. (1985). Protoplast fusion in Bacillus stubtilis and its consequences. In Molecular Biology of Bacilli, vol. II, pp. 110-146. Edited by D. Dubnau. New York: Academic Press.

9. Le Dérout, J., Thaler, D. S., Guillén, N. \& Hirschbein, L. (1992). The spo0 $A$ gene is implicated in the maintenance of non-complementing diploids in Bacilius subtilis. Mol Microbiol 6, 1495-1505.

10. Levi-Meyrueis, C. \& Sanchez-Rivas, C. (1984). Complementation and genetic inactivation: two alternative mechanisms leading to prototrophy in diploid bacterial clones. Mol \& Gen Genet 196, 488-493.

11. Levi-Meyrueis, C., Sanchez-Rivas, C. \& Schaeffer, P. (1980). Formation de bactéries diploides stables par fusion de protoplastes de Bacillus subtilis et effet de mutations recsur les produits de fusion formés. CR Acad Soi Par 291D $67-70$.

12. Lopez, F., Guillen, N. \& Hirschbein, L. (1986). Further characterization of the inactive chromosome from Bacillus subtilis stabilized non-complementing diploids. In Bacillus: Molecular Genetics and Biotechnology Applitations, pp. 73-86. Edited by A. T. Ganesan \& J. A. Hoch. New York: Academic Press

13. Rubinstein, C., Coso, O. A., Ruzal, S. M. \& SanchezRivas, C. (1993). Anti-SOS effects induced in B. subtilis by a $\phi 105$ mutant prophage. Arch Microbiol (in press).

14. Sanchez-Rivas, C. \& Garro, A. (1979). Bacterial fusion assayed by a prophage complementation test. / Bacteriol 137, 1340-1345.

15. Sanchez-Rivas, C., Levi-Meyrueis, C., Lazard-Monier, F. \& Schaeffer, P. (1982). Diploid state of phenotypically recombinant progeny arising after protoplast fusion in Bacillus subtilis. Mol do Gen Genet 188, 272-288.

16. Schaeffer, P., Cami, B. \& Hotchkiss, R. D. (1976). Fusion of bacterial protoplasts. Proc Natl Acad Si USA, 73, 2151-2155.

\section{No experimental support for diploidy and chromosomal silencing}

In their letter (above), C. Sanchez-Rivas and C. Levi-Meyrueis take as granted that the published evidence in favour of the diploidy of Bacillus subtilis exfusants is compelling and propose to rediscuss some specific experiments already extensively dealt with by us (4). While being prepared to enter into discussion, we feel that a detailed refutation of the con- 
clusions drawn from - in most instances irreproducible experiments [see Discussion in (4)] will not advance the debate.

We note that Sanchez-Rivas and LeviMeyrueis do not challenge our main new data i.e. (i) the mixed nature of exfusant colonies, which exhibit the so-called diploid phenotype (ii) the inadequacy of purification methods used in all previous experiments, and finally (iii) the validity of our reconstruction experiments with mixtures of whole cells. These experiments and their conclusion [i.e. that segregation of various parental and recombinant phenotypes from exfusants is due to the mixed nature of the original colonies and to transformation between various cells and possibly L-forms (P. M. Hauser, unpublished] are not invalidated by any newer results. Therefore, we believe that our criticisms of the concepts of diploidy and chromosome silencing remain valid.

We consider that the most plausible explanation proposed for the behaviour of the exfusants (4) is as follows. Multigenomic zygotes, obtained by protoplast fusion, allow recombination, as well as expression of all of the genomes. As long as such protoplasts or possible $\mathrm{L}$-forms are able to grow in the absence of cell wall regeneration, complementation would be possible. However, cell wall regeneration seems to preclude the maintenance of more than one genome and thus of complementing diploids. The experiments of C. Karmazyn-Campelli and others (3), perfectly in line with this interpretation, were interpreted in favour of a correlation between resumption of peptidoglycan synthesis and chromosomal silencing.

The brief summary and assessment of previous results by Sanchez-Rivas and LeviMeyrueis is revealing and hardly requires further comment. For instance, in a single paragraph of their letter, we read 'Abundant experimental data support the diploidy of all fusion types', as well as 'Every previous worker in this field has searched for the usual signs of diploidy without success'. It is stated that even increased amounts of DNA could not be detected. Would this mean that silencing of a chromosome prevented its acid hydrolysis and detection of diphenylamine-staining material? It is further stated that diploidy was demonstrated by restoring the transforming activity of the silenced chromosome. The presence of identical values in two distinct papers [Table 3 in (1); Table VII in (2)] confirm that the evidence is based on results of a single experiment which we were never able to reproduce.

Inspection of the statements of LeviMeyrueis and Sanchez-Rivas reveals that there does not seem to be much of a 'controversy' as far as the data on exfusants is concerned; any controversy concerns only the belief as to their meaning. Our experiments (4) fully account for the 'abundant experimental data which support the so-called diploidy of all fusion types' (see above), and have the advantage of not requiring an occult mechanism reducing the putative silent chromosome to an immaterial entity.

In conclusion, if a true diploid clone with a silenced chromosome could be obtained, it would provide an invaluable tool. However, until such a construct is available, experiments on $B$. subtilis protoplast fusion provide neither evidence for chromosomal silencing in prokaryotes, nor arguments for an evolutionary step in the passage from pro- to eukaryote. The latter conclusions will have to be abandoned, due to lack of unequivocal experimental support.

Philippe M. Hauser ${ }^{1}$ and Dimitri Karamata $^{2 *}$

${ }^{1}$ Chemical Pathology Unit, University of Oxford, South Parks Road, Oxford OX1 3RE, UK.

${ }^{2}$ Institut de génétique et biologie microbiennes, Rue César-Roux 19, 1005 Lausanne, Switzerland.

* For correspondence. Tel: +4121206075 Fax: +4121206078.

1. Bohin, J. P., Ben Khalifa, K., Guillén, N., Schaeffer, P. \& Hirschbein, L. (1982). Phenotypic expression in vivo and transforming activity in vitro: two related functions of folded bacterial chromosomes. Mol \& Gen Genet 185, 65-68. 2. Guillén, N., Amar, M. \& Hirschbein, L. (1985). Stabilized non-complementing diploids $(\mathrm{Ncd})$ from fused protoplasts products of $B$. subtilis. EMBO /4, 1333-1338.

3. Karmazyn-Campelli, C., Savelli, B., Rogers, H. J. \& Schaeffer, P. (1985). Inverse relationship between formation of phenotypically recombinant clones and cell wall regeneration after fusion of Bacillus subtilis protoplasts. J Gen Microbiol 131, 1635-1638.

4. Hauser, P. M. \& Karamata, D. (1992). Ploidy of Bacillus subtilis exfusants: the haploid nature of cells forming colonies with biparental or prototrophic phenotypes. J Gen Microbiol 138, 1077-1088 